RESEARCH ARTICLE

\title{
Demographic Features of COVID-19 in Two Waves in A Single Center
}

\author{
Rishav Mukherjee ${ }^{1}$, Dharitri Chaudhuri ${ }^{2}$, Arup Kumar Mitra ${ }^{1}$, Partha Guchhait ${ }^{3}$, Subhrojyoti Bhowmick ${ }^{4}$, \\ Satadal Das ${ }^{3}$, Bhaskar Narayan Chaudhuri ${ }^{3}$ \\ ${ }^{1}$ Department of Microbiology, St. Xavier's College, Kolkata, West Bengal, India \\ ${ }^{2}$ Department of Biotechnology, St. Xavier's College, Kolkata, West Bengal, India \\ ${ }^{3}$ Department of Microbiology \& Molecular Biology, Peerless Hospitex Hospital \& Research Center Ltd., Kolkata, West \\ Bengal, India \\ ${ }^{4}$ Department of Clinical Research and Academics, Peerless Hospitex Hospital \& Research Center Ltd., Kolkata, West \\ Bengal, India
}

\section{ABSTRACT}

Objectives: This study was undertaken to understand commonly affected age groups, the prevalence of asymptomatic cases, and vaccine efficacy in Kolkata-centric COVID-19 patients.

Methods: A retrospective analysis of COVID-19 patients' data from May 2020 to July 2021 from Peerless Hospitex Hospital \& Research Center Ltd., a tertiary care private hospital of Kolkata, West Bengal, was done.

Results: A total of 67,121 samples were tested for COVID-19 between May 2020 and July 2021, with the COVID-19 positivity rate of $23.8 \%$ noted over this period. A direct correlation was found between the number of tests conducted and the positivity. The first wave of the pandemic was observed to be from May to December 2020, while the second wave began from April 2021 and declined by July 2021. This correlated with the first and second waves observed in India. The first wave mainly affected the elderly population, while the second wave more affected the younger and middle-aged population. The percentage of asymptomatic patients increased during the second wave compared to the first wave. By comparing the percentage of vaccinated individuals who tested COVID-19 positive following Covaxin vs. Covishield vaccination, although after the first dose, Covishield was observed to be more effective than Covaxin, after the second dose, efficacies of Covaxin and Covishield were not found to differ significantly.

Conclusion: The pattern of COVID-19 cases in Kolkata is similar to the National pattern; however, in the second wave, younger and middle-aged persons, females, and asymptomatic cases were significantly more than the first wave. J Microbiol Infect Dis 2021; 11(4):174-182.

Keywords: COVID-19, demography, vaccine efficacy

\section{INTRODUCTION}

The first outbreak of COVID-19 occurred in December 2019 when a novel coronavirus, now named SARS-CoV-2 (Severe Acute Respiratory Syndrome Coronavirus-2), caused acute respiratory distress syndrome (ARDS) with high mortality and morbidity rates [1-3].

Common symptoms include fever, sore throat, dry cough, dyspnoea, fatigue, myalgia, headache, and weakness. Anosmia or ageusia may be the only presenting symptom in some cases [4]. Uncontrolled production of proinflammatory mediators results in Acute Respiratory Distress Syndrome (ARDS) and cytokine storm [5].

India reported its first case of COVID-19 on 30th January 2020 when a 20-year-old medical student who had returned from Wuhan University tested COVID positive [6]. An 18-

Correspondence: Dr. Bhaskar Narayan Chaudhuri, Department of Microbiology \& Molecular Biology, Peerless Hospitex Hospital \& Research Center Ltd., Kolkata, West Bengal, India 
year-old student who had returned from the United Kingdom tested COVID positive on $17^{\text {th }}$ March 2020 - the first COVID-19 case of West Bengal. After that, the disease spread at an exponential rate, killing people due to direct infection with the virus and heightened immune response of the body against it and due to economic collapse when developing countries like India suffered from unemployment and hunger. As of $19^{\text {th }}$ November 2021, there have been a total of 255,324,963 COVID positive cases and $5,127,696$ deaths due to COVID-19 infection [Covid 19 live dashboard, WHO].

Mass vaccination campaigns have commenced worldwide to prevent the infection and transmission of SARS-CoV-2. Currently, the vaccines authorized for use globally are Pfizer-BioNTech, Moderna, Johnson \& Johnson/ Janssen, Oxford-AstraZeneca, Covaxin. India started its vaccination program on 16th January 2021. Two vaccines are predominantly being administered in India to prevent the spread of SARS-CoV-2 Covishield, the Adenovirus vector-based vaccine from Oxford University and AstraZeneca, UK; and Covaxin, the indigenous inactivated virus vaccine manufactured by Bharat Biotech.

However, the emergence of novel variants of the virus due to mutations in the spike protein, which leads to a higher infectivity rate and ability to infect even vaccinated individuals, still poses a severe threat. Four major variants of concern (VOCs), i.e., B.1.1.7, B.1.1.351, P.1, B.1.617.2 have been identified by the Indian SARS-CoV-2 Genome Consortia (INSACOG), and those are associated with increased virulence and reduced efficacy of vaccines [7]. Of these 4 VOCs, the B.1.617.2, also called the Delta variant, is believed to spread the fastest. Furthermore, it has been demonstrated through recent in-vitro studies that sera from Pfizer or AstraZeneca vaccinated individuals is less effective in neutralizing the Delta variant compared to the Alpha variant (B.1.1.7) [8].

Aims of the study:

This study aimed to observe the COVID-19 test positivity fluctuation in this hospital during the first and second wave along with the agewise and gender-wise distribution of COVID-19 positive cases during the two waves; the proportion of symptomatic vs. asymptomatic positive cases, and to determine the efficacy of Covaxin and Covishield vaccines.

\section{METHODS}

The study was done after receiving approval from the Institutional Ethics Committee of Peerless Hospitex Hospital and Research Center Ltd. (Letter No.: PHH \& RCL/CREC/FM02 dated 9th September 2021). This retrospective study was conducted by collecting data of people who came to test for COVID-19 from 1st May 2020 to 31st July 2021 at Peerless Hospital, Kolkata, from the institution's database. Only patients from the Kolkata district were filtered, and the others were excluded from the study. The demographic characteristics of all samples were noted. COVID-19 positive samples were selected and compared with the total samples tested. The percentage positivity for each month was calculated and plotted to determine the differences between the first and second waves of the pandemic.

The population was divided into four different age groups based on the phase-wise target age groups for vaccination, which started from March 2021 for the general population of India. These groups were $<18$ years, between 18 and 45 years, between 45 and 60 years, and $>60$ years. The percentage of COVID-19 positive individuals for each age group was calculated, and a comparison of these age group wise COVID-19 positive percentages for the years 2020 and 2021 was determined. In addition, the numbers of male and female COVID-19 positive individuals for each age group were calculated and compared. Subsequently, the number of COVID-19 positive patients who were symptomatic and the number of COVID-19 positive patients who were asymptomatic at the time of testing were calculated, and the percentages of symptomatic and asymptomatic COVID-19 patients in 2020 and 2021 were compared.

Lastly, the vaccination status was studied by comparing the two doses separately for Covishield and Covaxin; their efficacies were studied by calculating the percentage of vaccinated individuals who tested COVID-19 positive.

All graphs were generated in MS-Excel. The Test statistics used were the 2-sample test for equality of proportions (Chi-square test), exact binomial test, and test for the correlation 
coefficient. Statistical analysis was done using statistical package $R$ version 3.5.3. The Chisquare test was used for determining the statistical significance of the results. A P-value of $<0.05$ was considered significant.

\section{RESULTS}

\section{The Total number of Kolkata positive tests:}

A total of 67,121 samples were tested in the Molecular Testing Laboratory of the Hospital from Kolkata, with the COVID-19 positivity rate of $23.8 \% \quad(n=15,949)$ noted for 15 months (May 2020 to July 2021). It was observed that when the number of positive cases was more, the number of tests conducted also rose simultaneously, and, on the other hand, when there was a dip in positivity, the number of tests conducted also waned (Figure 1). Correlation coefficient between no. of positivity and no. of tests $=0.862987, \mathrm{P}-$ Value $=3.439 \mathrm{e}-$ $05(<0.05)$.

\section{Observations in the first wave:}

A line diagram (Figure 2) showed a monthwise percentage of COVID-19 positivity data of Kolkata during the study period. The first wave started in May 2020 and persisted until December 2020, with the highest peak (35.46\%) observed in October 2020. A stationary phase was noted between January to March 2021 when the positivity rate varied between 2 and $5 \%$ but never touched the baseline. A comparative analysis of the percentage of COVID-19 positivity between 2020 and 2021 was determined among four different age groups depicted by a bar diagram in Figure 3. Comparison between male and female COVID-19 positive individuals revealed that more number $(n=9253$ out of 15949 positive cases, i.e., $58.02 \%)$ of males $(95 \% \mathrm{Cl}$, 57.63-58.41) were affected than females $(n=6696$, i.e., $41.98 \% ; 95 \mathrm{Cl}, 41.59-42.31)$, during the study period. Age group-wise analysis in the first wave shown in Figure 4, male preponderance was mainly observed in > 60 -year individuals $(33.63 \%$ in males vs. $27.98 \%$ in females. In the first wave, $59.17 \%$ $(n=4450)$ of 7521 COVID-19 positive patients were symptomatic at the time of testing, whereas $40.83 \%$ were found to be asymptomatic.

\section{Observations in the second wave:}

The second wave commenced in late March 2021 and is yet to touch the baseline. The highest positivity rate $(44.94 \%)$ was observed in May 2021, following which it dropped drastically for the next two months to $3.17 \%$ in July 2021. Age-group-wise comparison of positive cases in the second wave is depicted in Figure 3. In the second wave, females were affected more in young adults and older adults, with a percentage positivity rate of $41.91 \%$ and $31.32 \%$, respectively. Male predominance was observed with $28.35 \%$ positivity in $>60$ age group individuals. P-value was significant $(<0.05)$ in all the above scenarios. A different scenario was observed in the second wave, with $53.34 \%$ of patients reporting asymptomatic at the time of testing and $46.67 \%$ of patients reporting symptomatic.

\section{Changing pattern from the first wave to second wave:}

The group younger than 18 years showed an increase in positivity from $2.75 \%$ in the first wave to $4.37 \%(95 \% \mathrm{Cl}, 4.37-4.83)$ in the second wave.

The group between 18 and 45 years showed a rise in positivity rate from $33.98 \%$ in the first wave to $40.13 \%$ in the second wave $(95 \% \mathrm{Cl}$, 39.60-41.66). Both of the occasions have significant changes $(p=<0.05)$. On the contrary, the other two groups showed a significant decline in the positivity rate $(p=$ $<0.05$ ). There was a male dominancy in the group of $>60$ years in the first wave. In the second wave, females were affected more in both young adult and older adult age groups. P-value was significant $(<0.05)$ in all the above scenarios. Asymptomatic cases were more in the second wave.

\section{Comparison of vaccine efficacy in terms of positive cases:}

Till July 2021, a total of 1744 patients received the first dose, and 828 patients got their second jab of either Covishield or Covaxin, since the inception of COVID vaccination in January 2021. Out of 1744 patients with the first vaccination dose, 567 (32.51\%) patients $(95 \% \mathrm{Cl}, 30.31-34.71)$ were COVID-19 positive by RT-PCR. However, the positivity rate declined significantly $(p<0.05)$ to $20.89 \%$ $(95 \% \mathrm{Cl}, 18.12-23.66)$, with 173 patients testing positive out of 828 patients after receiving the second dose of vaccination.

The efficacies of Covishield and Covaxin were studied by calculating the percentage of vaccinated individuals who tested COVID-19 
positive after the first dose and the second dose, as depicted in Figure 5. Positivity rate following the 1st dose was found to be less with Covishield $(30.30 \%)$ as compared to Covaxin (47.16\%); $p=5.665 \mathrm{e}-07(<0.05)$. In terms of the development of infection after the second dose vaccination, Covaxin showed better efficacy than Covishield (Covaxin: $15.13 \%$ vs. Covishield: $21.86 \%$ ). However, this difference was not statistically significant $(p=0.121)$.

Table 1. Comparison between symptomatic and asymptomatic COVID-19 positive cases (2020 vs 2021).

\begin{tabular}{|l|c|c|}
\hline Year & Symptomatic COVID-19 & Asymptomatic COVID-19 \\
\hline 2020 (First wave) & $59.17 \%$ & $40.83 \%$ \\
\hline 2021 (Second wave) & $46.67 \%$ & $53.34 \%$ \\
\hline
\end{tabular}

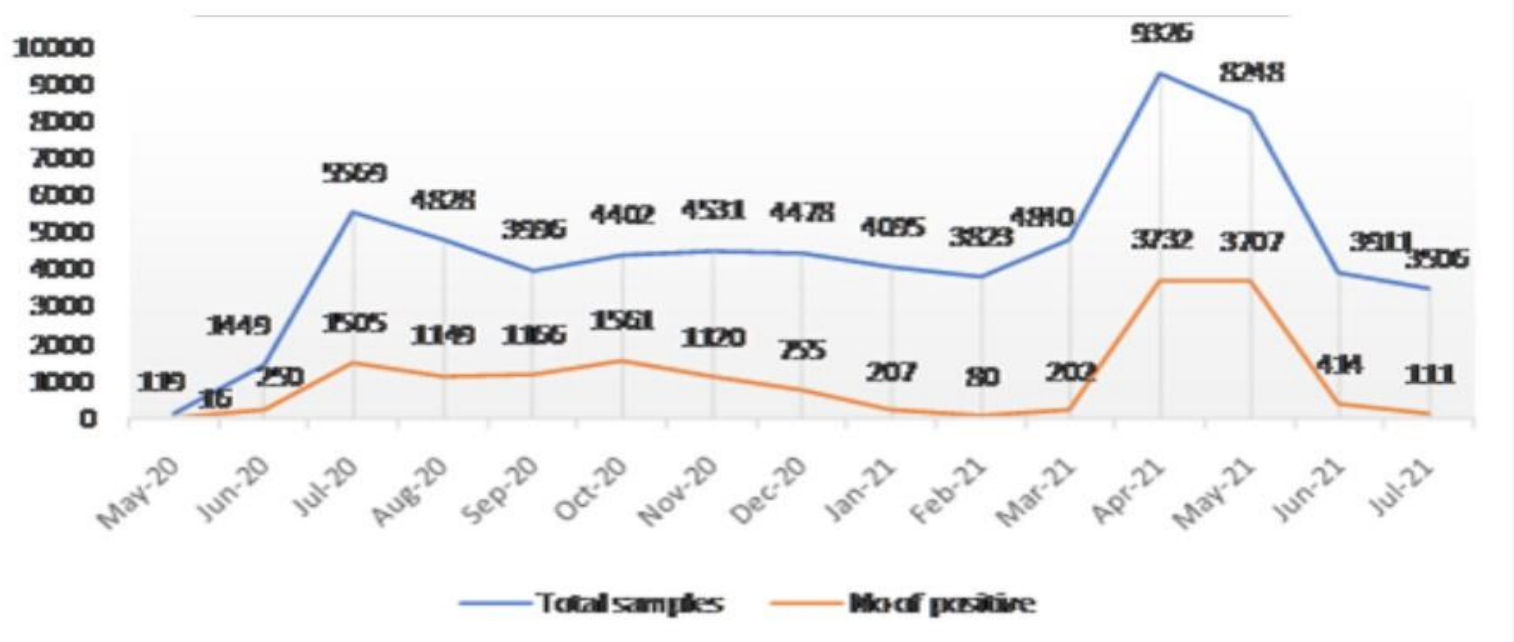

Figure 1. The total numbers of tests conducted vs. confirmed positive cases (May 2020-July 2021).

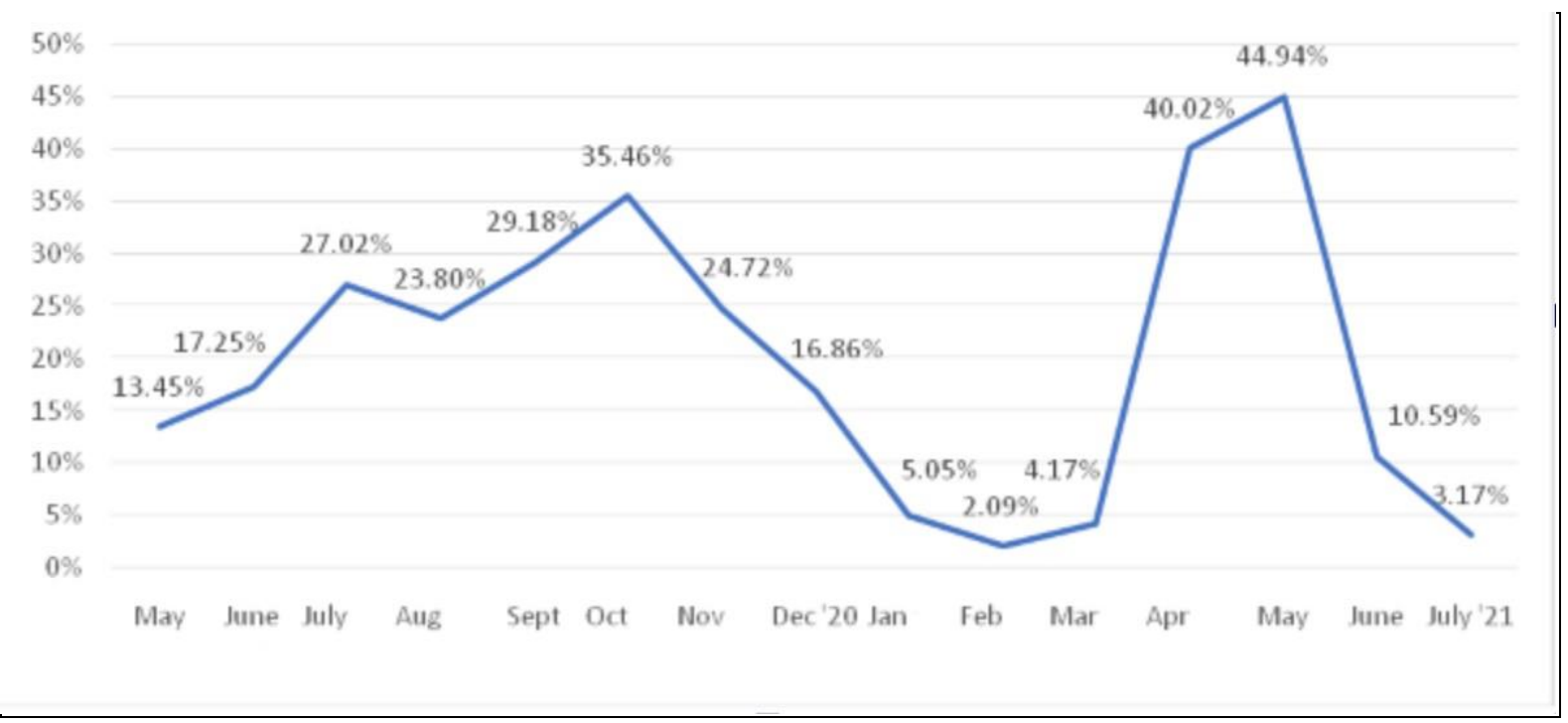

Figure 2. The COVID-19 test positivity rate in Kolkata from May 2020 to July 2021. 


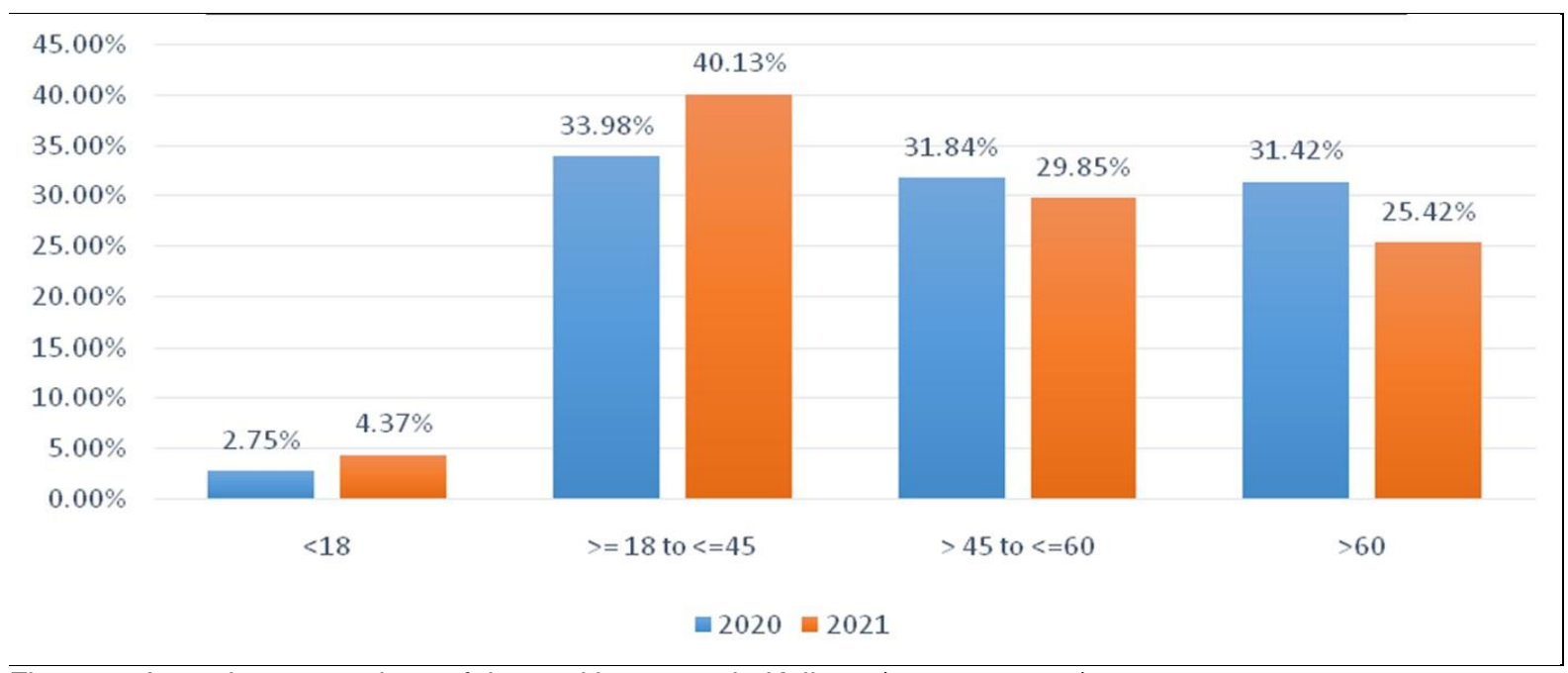

Figure 3. Age-wise comparison of the positive cases in Kolkata (2020 vs. 2021).

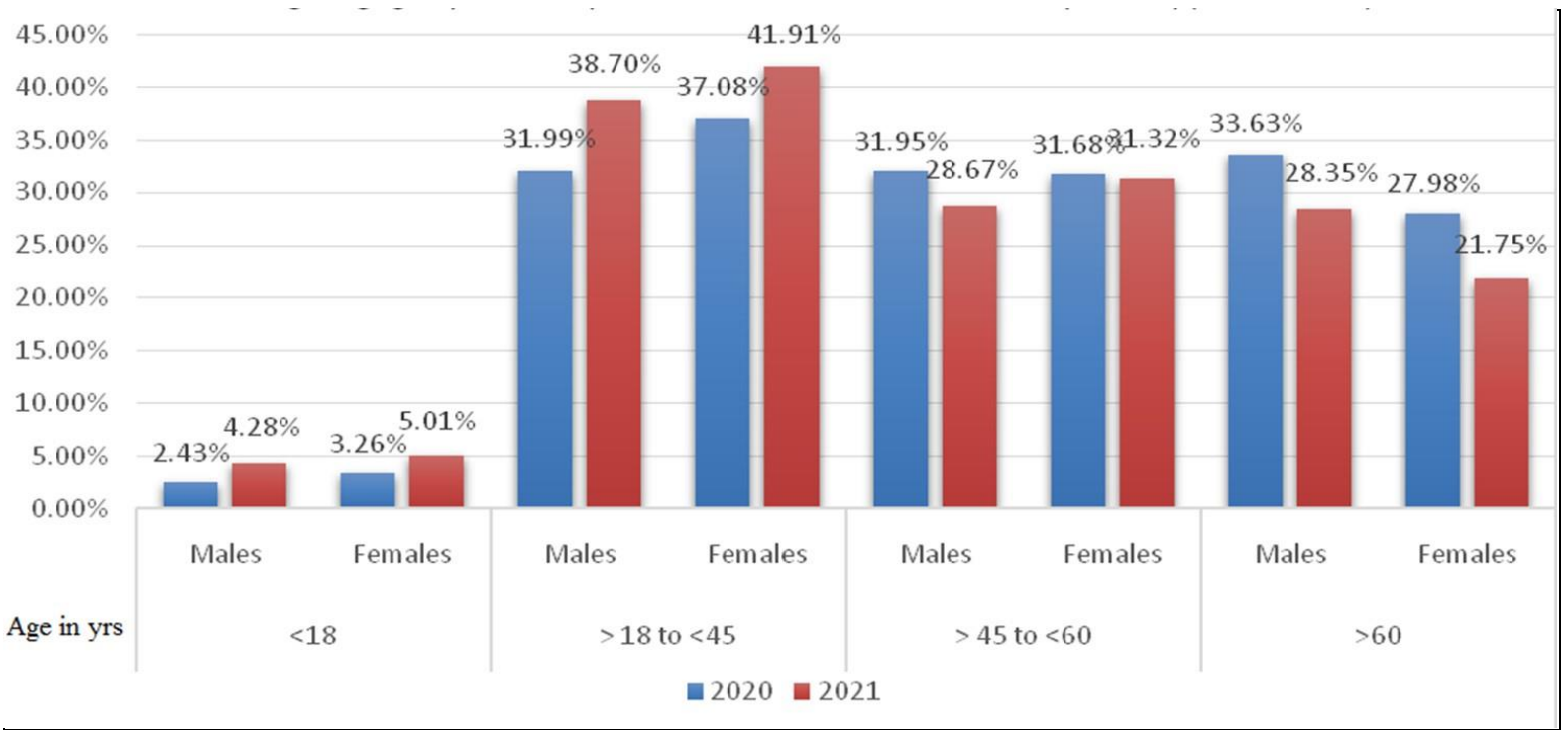

Figure 4. Age group based comparison between gender in term of COVID-19 positivity (2020 vs. 2021)

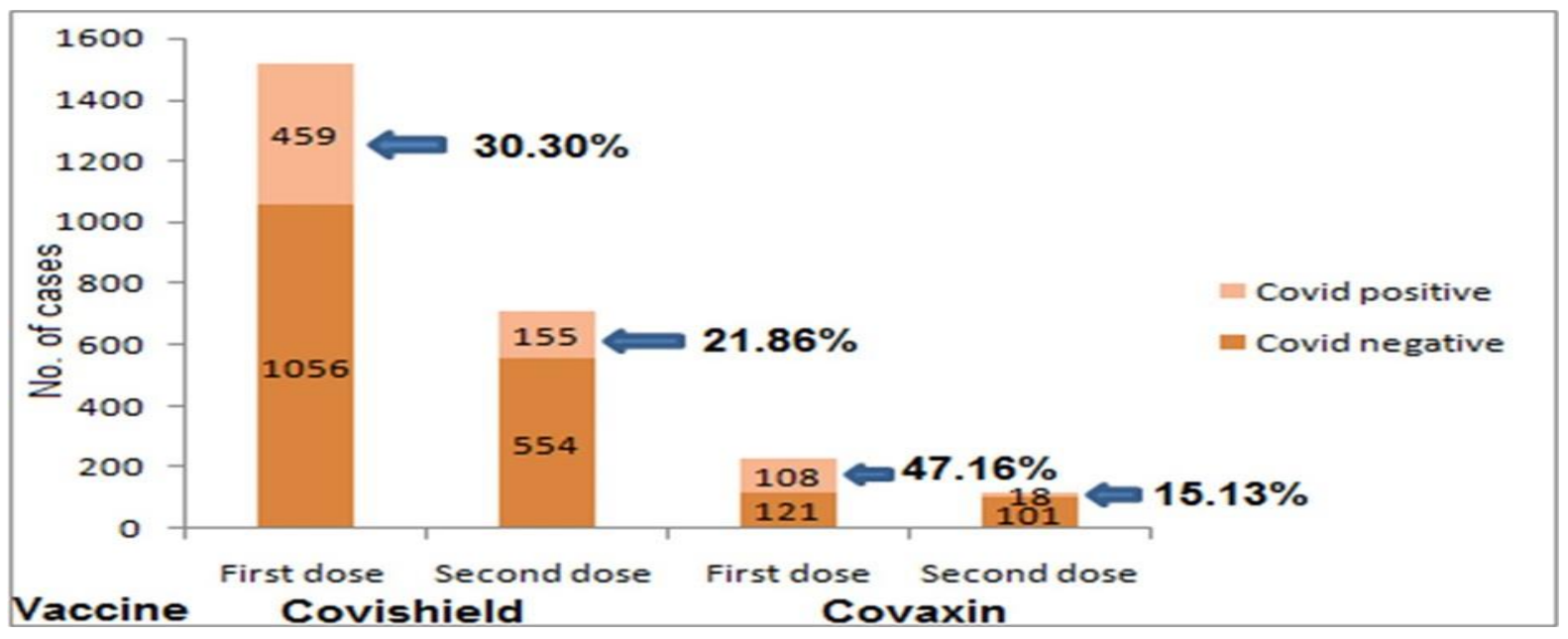

Figure 5. Comparison of vaccine efficacy in terms of positive cases. 


\section{DISCUSSION}

To the best of our knowledge, this is the first such study concerning patient demographics and COVID vaccine comparison conducted in our part of the country. This retrospective study finds that the positivity percentage increases when the number of tests goes up, i.e., there is a direct relation between the positive cases and the number of tests conducted. The testing number could be influenced by several factors such as restrictions during the lockdown, public unawareness. Nevertheless, on the other hand, people become complacent about wearing masks and maintaining physical distancing, which may lead to a rise in the number of symptomatic individuals, psychological factors like getting in contact with positive individuals, and fear psychosis.

The maximum positivity was in October $(35.46 \%)$ in the first wave, which can be attributed to the festive season in Bengal. In 2021, the COVID situation was under control for the first three months - January to March. Similarly, a study by Sharma in Delhi also indicated that the test positivity rate was highest from October 2020 to mid-November 2020 with a subsequent steep declining trend, followed by a persistently low case burden lasting until the first week of March 2021 [9]. It is important to note that it was during this time (30th December 2020) that Kolkata reported its first case of mutant virus strain [Times of India]. After that, there was a steep rise in positivity, which heralded the second wave in April 2021 (40.02\%) and reached its peak in May 2021 (44.94\%), which also supports the study by Sharma et al., which shows the exponential growth of positivity from April 2021 onwards [9]. Thus, the findings of this study are consistent with the Indian scenario, as a direct correlation was observed between COVID-19 test positivity fluctuation in this area vis-a-vis country-wide fluctuation in the number of cases. Mainly the double mutant 'Delta' strain (B.1.617) and the triple mutant 'Bengal Strain' (B.1.618) were responsible for the increase of positivity during the second wave $[10,11]$. The Delta strain along with the triple mutant 'Bengal strain' (a deletion of two changes in spike protein; deletion of $\mathrm{H} 146$ and $\mathrm{Y} 145$; and mutation in E484K and D614G in the $S$ glycoprotein) show higher transmissibility and bypass antibody response induced from natural infection or vaccine $[9,11]$. Genome sequencing should be considered on a caseto-case basis, as per the criteria laid down by the Indian SARS- CoV-2 Consortium on Genomics, for a better understanding of mutants in a geographic area and to get a clue if there is the emergence of any new strain, which could turn into a Variant of concern and leading to the third wave.

Many previous studies showed that susceptibility to COVID-19, along with pathogenic severity, is more in the older people, who have co-morbidities like hypertension, diabetes, and structural lung diseases. [12-15]. However, interestingly, the age group analysis in our study reveals that compared to 2020 (first wave), the positivity percentage for the older population decreased in 2021 (second wave). The most probable reason for this is vaccination, which started for the general population in India from March 2021 for above 60 years and from 1st April 2021 for above 45 years age groups. Furthermore, this could also be the reason why the younger age groups showed an increase in the percentage of positivity during the second wave as they were not covered by vaccination.

Although clinical characteristics suggest both males and females are equally likely to get COVID-19 [16], clinical outcomes in various studies suggest otherwise. Some studies show males are predominantly more affected than females, while others show the opposite [17]. It can be suggested that higher $\mathrm{ACE}_{2}$ expression and activity in males than females may contribute to sex differences in COVID-19 infection and fatality. Many studies have noted that $\mathrm{ACE}_{2}$ activity is higher in males than in females $[18,19]$.

In the gender-based analyses of four different age groups in our study, the males showed higher positivity $(58.02 \%)$ than females $(41.98 \%)$ during these 15 months in general, but in the second wave, the positivity rate decreased, with an opposite effect in females particularly in the younger age groups. It may be true that females encounter lesser severity in COVID infection than males [20] as they have higher cell-mediated and humoral immune responses [21]. However, the results can be explained by a study performed in Uttar 
Pradesh and Bihar of India by Pinchoff et al.. This study revealed that women were less likely to practice COVID-appropriate behavior because they could not correctly identify the main COVID-19 symptoms due to challenges in accessing information or receiving less accurate information of COVID-19 symptoms [22]. Another probable reason may be gender discrimination in society as regards vaccination.

The increase of asymptomatic patients in the second wave may mean more awareness among the people during the second wave, prompting them to go for testing early, when the infection was still incubating. Also, several patients did not divulge their symptoms while presenting themselves for testing, especially during the second wave. This result also supports the findings of a Hong Kong study where it was observed that asymptomatic individuals were more during the second and third waves than during the first [23].

In a nutshell, the main demographic features captured in this study showed a shift in the mean age of COVID-19 positive cases from the elderly age group to the younger age group; a proportionate increase of female positivity; and an increase in asymptomatic positive cases during the second wave in comparison to the first wave.

Immunity acquired from COVID-19 infection or vaccination is not permanent, and re-infection (breakthrough infection) after vaccination is common [24-26]. Though Singh et al. [26] did a study about breakthrough COVID-19 infection post-vaccination, our study is unique in that the sample size is greater than the previous study. Our study shows that in the post-vaccination scenario, however, Covishield was more effective after the first dose. Still, after the second dose, both Covishield and Covaxin were equally effective. Covishield is a recombinant replication-deficient chimpanzee adenovirus-vectored vaccine encoding only SARS-CoV-2 spike antigen, and Covaxin is a ß-propiolactone inactivated whole virion vaccine having all structural SARS-CoV-2 antigens [28]. Theoretically, therefore, as the new variants have mutations in the spike proteins, Covishield (targeted against the spike protein) is less likely to be effective than Covaxin (targeted against the whole virion). However, this could not be established through our study. Though in some studies, it was observed that seropositivity is higher following Covishield vaccination than Covaxin, both after1stdose [27] and second dose [28], but no direct study proves that the humoral antibody response has a direct relationship with the efficacy of the vaccine. Nevertheless, it must be acknowledged that a greater number of people got Covishield $(n=2,224)$ than Covaxin $(n=348)$. In addition, the period between 2 doses of Covishield is greater than that for Covaxin. Thus, the limitation of our study is that the sample size of the second dose of Covaxin $(n=119)$ is by far less compared to that of Covishield $(n=709)$. However, despite the simplicity of our findings, there are some interesting insights in the first two waves of COVID-19 which may be useful.

There are also some other limitations in this study. Restrictions influence the testing number during lockdown periods. In this study, detailed clinical COVID-19 data could not be retrieved. The co-morbidities were not collected in the Specimen Referral Forms as efficiently during the second wave due to lack of declaration by the patients, against those were captured during the first wave. Due to this limitation, this study could not include the comparative study of positivity rates in different age groups concerning co-morbidities during the first and second waves. Again the results are based on past data, and they may not reflect the present scenario.

Conclusion: COVID-19 cases in Kolkata are similar to the overall pattern in India; however, in the second wave, young and middle-age groups, females, and asymptomatic cases were significantly more than the first wave. Again Covishield vaccine was found more effective after the first dose, but after the second dose, they are almost similar in efficacy.

\section{ACKNOWLEDGMENTS}

Author Contributions: AKM, PG and BNC conceptualized and designed the study. RM and DC carried out the data analysis. RM and DC wrote the manuscript. The manuscript was critically reviewed and rewritten by BNC, PG, $A K M, S D$ and $S B$. SB and SD helped in planning and provided their valuable suggestions. All the authors have gone through the manuscript and approve the submission. 
Declaration of conflicting interest: The author(s) declare no potential conflicts of interest concerning this article's research, authorship, and/or publication.

Financial disclosure: No financial support was received for this study.

\section{REFERENCES}

1. Singhal T A Review of Coronavirus Disease2019 (COVID-19). Indian J Pediatr 2020; 87(4):281-286.

2. Ksiazek TG, Erdman D, Goldsmith CS, Zaki SR, Peret $\mathrm{T}$, Emery $\mathrm{S}$ et al. A novel coronavirus associated with severe acute respiratory syndrome. N Engl J Med 2003; 348(20):195366.

3. Hoffmann M, Kleine-Weber $H$, Schroeder $S$, Krüger N, Herrler, Erichsen S, et al. SARS-CoV2 cell entry depends on ACE2 and TMPRSS2 and is blocked by a clinically proven protease inhibitor. Cell 2020;181(2): 271-280.

4. Mao R, Qiu Y, He JS, Yu TJ, Hua LX, Liang J et al. Manifestations and prognosis of gastrointestinal and liver involvement in patients with COVID-19: a systematic review and metaanalysis. Lancet Gastroenterol Hepatol 2020; 5(7):667-678.

5. Felsenstein $S$, Herbert, J A, McNamara P S, Hedrich C M. COVID-19: Immunology and treatment options.Clin Immunol 2020; 215:108448.

6. Andrews M A, Areekal B, Rajesh K R, Krishnan $J$, Suryakala R, et al. First confirmed case of COVID-19 infection in India: A case report. The Indian Journal of Medical Research 2020; 151(5), 490-492.

7. INSACOG: Indian SARS-CoV-2 Genomics Consortium.

8. Planas D., Veyer D, Baidaliuk A. Reduced sensitivity of SARS-CoV-2 variant Delta to antibody neutralization. Nature 2021; 596, 276280.

9. Sharma N, Sharma P, Basu S, Bakshi R, Gupta $\mathrm{E}$, Agarwal $\mathrm{R}$ et al., Second wave of the Covid19 pandemic in Delhi, India: high seroprevalence not a deterrent? med Rxiv 2021; 09.09.21263331.

10. Sahoo JP, Mishra AP, Samal KC. Triple Mutant Bengal Strain (B. 1.618) of Coronavirus and the Worst COVID Outbreak in India. Biotica Research Today 2021;3 (4):261-5.

11. Vakili S, Savardashtaki A, Sheida J, Tabrizi R, Nematollahi $\mathrm{M} \mathrm{H}$, Jafarini $\mathrm{M}$ et al., Laboratory Findings of COVID-19 Infection are Conflicting in Different Age Groups and Pregnant Women: A Literature Review. Arch Med Res 2020; 51(7):603-607.

12. Bonanad C, Garcia-Blas S, TarazonaSantabalbina F, Sanchis J, Bertomeu-Gonzalez
$V$, Facila $L$ et al. The Effect of Age on Mortality in Patients with COVID-19: A Meta-Analysis with 611,583 Subjects. J Am Med Dir Assoc 2020; 21(7):915-918.

13. Parthymou A, Habeos E, Habeos G I, Deligakis A, Livieratos E, Marangos M et al., Sars-Cov-2 antibody titer 3 months post-vaccination is affected by age, gender, smoking and vitamin D. med Rxiv 2021; 09.01.21262913.

14. Han Y, Luo Z, Zhai W, Zheng Y, Liu H, Wang Y, et al. Comparison of the clinical manifestations between different age groups of patients with overseas imported COVID-19. PLoS ONE 2020; 15(12): e0243347

15. Jin JM, Bai P, He W, Wu F, Liu XF, Han DM, et al. Gender differences in patients with COVID19: focus on severity and mortality. Front Public Health 2020; 8:152.

16. Mukherjee, S., Pahan, K. Is COVID-19 Gendersensitive?. J Neuroimmune Pharmacol 2021; $16,38-47$.

17. Cai G, Bosse Y, Xiao F, Kheradmand F, Amos $\mathrm{Cl}$ Tobacco smoking increases the lung gene expression of ACE2, the receptor of SARS-CoV2.2020, Am J Respir Crit Care Med 2021;15571559.

18. Gargaglioni LH, Marques DA, Let's talk about sex in the context of COVID-19. J Appl Physiol 2020; 128:1533-1538.

19. Ruggieri A, Anticoli S, D'Ambrosio A, Giordani L, Viora $M$, The influence of sex and gender on immunity, infection and vaccination. Ann Ist Super Sanita 2016; 52:198-204.

20. Fish $E N$. The X-files in immunity: sex-based differences predispose immune responses. Nat Rev Immunol 2008; 8:737-744.

21. Pinchoff J, Santhya K, White C, Rampal S, Acharya R, Ngo TD, Gender specific differences in COVID-19 knowledge, behavior and health effects among adolescents and young adults in Uttar Pradesh and Bihar, India. PLoS ONE 2020; 15(12): e0244053.

22. Chua G, Chih Wang JS, LAM I et al., Clinical characteristics and transmission of COVID-19 in children and youths during 3 waves of outbreaks in Hong Kong. JAMA 2021; 4(5):e218824.

23. Mukherjee A, Anand T, Agarwal Anup. SARSCoV-2 re-infection: development of an epidemiological definition from India. Epidemiol. Infect 2021; 149.

24. Philomina J B, Jolly B, John N, Bhoyar RC, Majeed N, Senthivel V,N et al. Genomic survey of SARS-CoV-2 vaccine breakthrough infections in healthcare workers from Kerala, India. J Infect 2021; 83(2):237-279.

25. Hacisuleyman E, Hale C, Saito Y. Vaccine Breakthrough Infections with SARS-CoV-2 Variants. N Engl J Med 2021; 384:2212.

26. Singh U B, Rophina M, Chaudhry R, Senthivel V, Bala K, Bhoyar R C et al. Variants of Concern responsible for SARS-CoV-2 vaccine breakthrough infections from India. J Med 
Virology

2021

https://onlinelibrary.wiley.com/doi/epdf/10.1002/j

mv. 27461

27. Singh A K, Phatak S P, Singh R, Bhattacharjee K, Singh NK, Gupta A et al. Antibody Response after First-dose of ChAdOx1-nCOV (Covishield TM®)and BBV-152 (Covaxin TM®) amongst Health Care Workers in India: Preliminary Results of Cross-sectional Coronavirus Vaccineinduced Antibody Titre (COVAT) study. med Rxiv 2021; 06.02.21258242

28. Singh A K, Phatak S P, Singh R, Bhattacharjee K, Singh NK, Gupta A et al.; Antibody Response after Second-dose of ChAdOx1-nCOV (Covishield $\mathrm{TM} \otimes$ ) and BBV-152 (Covaxin TM®) among Health Care Workers in India: Final Results of Cross-sectional Coronavirus Vaccineinduced Antibody Titre (COVAT) study. med Rxiv 2021; 06.02.21258242 